\title{
Diastolic Dysfunction in Liver Cirrhosis: Prognostic Predictor in Liver Transplantation?
}

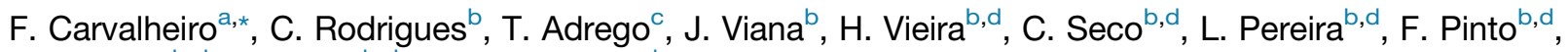 \\ A. Eufrásio $^{b, d}$, C. Bento ${ }^{b, d}$, and E. Furtado ${ }^{d}$

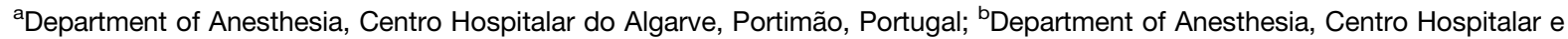 \\ Universitário de Coimbra, Coimbra, Portugal; ' $P u b l i c$ Health Unit of Baixo Mondego, Coimbra, Portugal; and ${ }^{\text {d} A d u l t ~ a n d ~ P e d i a t r i c ~ L i v e r ~}$ \\ Transplant Unit, Centro Hospitalar e Universitário de Coimbra, Coimbra, Portugal
}

\begin{abstract}
Background. Patients with liver cirrhosis may develop cirrhotic cardiomyopathy (CC), characterized by blunted contractile responsiveness to stress, diastolic dysfunction (DD), and electrophysiological abnormalities. It may adversely affect the long-term prognosis of these patients.

Methods. We conducted a retrospective analysis of patients undergoing liver transplantation (LT) for cirrhosis from January 2012 to June 2015. We analyzed demographic characteristics, the etiology of cirrhosis, Child-Pugh and Model for End-Stage Liver Disease (MELD) scores, the corrected QT (QTc) interval in the preoperative period, diastolic and systolic dysfunction, mortality and survival, and duration of mechanical ventilation and vasopressor support in the post-LT period. These variables were compared with diastolic dysfunction and prolongation of QTc, with the use of chisquare, Fisher, and Mann-Whitney $U$ tests.

Results. The study included 106 patients, $80.2 \%$ male and overall average age 54.83 years. The median MELD score was 16, and Child-Pugh class C in 55.4\%. Prolonged QTc interval before LT was present in $19 \%$ and DD in $35.8 \%$ of patients. QTc before LT or DD did not vary significantly with MELD or Child-Pugh score.

Conclusions. The patients in the pre-LT period presented with a significant incidence of DD, which can predispose them to adverse cardiac events. The presence of DD correlates with mortality after LT in patients with hepatic cirrhosis.
\end{abstract}

$\mathbf{P}$ ATIENTS with liver cirrhosis may develop cirrhotic cardiomyopathy (CC), characterized by a blunted contractile responsiveness to stress and/or altered diastolic relaxation with electrophysiologic abnormalities in the absence of known cardiac disease [1].

The hyperdynamic circulation in cirrhosis was described 60 years ago [2] and is characterized by decreased peripheral resistance, increased cardiac output and stroke volume, and low systolic arterial blood pressure. Cardiac dysfunction in cirrhotic patients was first described in patients with alcoholic cirrhosis and was attributed to the direct effect of alcohol. Indeed, alcohol was not correlated with the etiology of cirrhosis [3].

The disease usually remains silent with near normal cardiac function unless the patients are exposed to stress.
Systolic function may be maintained at rest, but physical or pharmacologic stress usually unmasks underlying systolic dysfunction in these patients [4].

Left ventricular diastolic dysfunction (DD) seems to be the first manifestation of $\mathrm{CC}$, because it usually appears before the features of systolic dysfunction [5]. The prevalence of DD in cirrhotic patients is $\sim 40 \%$ and is not related to the etiology and stage of liver disease, although its severity correlates with the degree of liver failure [5]. Other

*Address correspondence to Filipa Carvalheiro, Department of Anaesthesia, Centro Hospitalar do Algarve, Sítio do Poço Seco, 8500-338 Portimão, Portugal. E-mail: anafcarvalheiro@gmail. com 
studies show that although DD is a frequent event in cirrhosis, it is usually of mild degree and does not correlate with severity of liver disease [6]. DD seems to be a negative prognostic factor for patients undergoing transjugular intrahepatic portosystemic shunt (TIPS) insertion and patients undergoing liver transplantation (LT) [5]. Cardiac failure has emerged as an important cause of mortality after LT and accounts for $7 \%-21 \%$ of deaths in the postorthotopic LT period [6].

Electrophysiologic abnormalities observed in cirrhosis include prolonged QTc interval, electromechanical dyssynchrony, and chronotropic incompetence [7]. Prolongation of QTc represents the main electrocardiographic (ECG) characteristic of CC. It is detected in $40 \%-50 \%$ of cirrhotic patients and is not related to the etiology of liver disease [5]. It is considered to be one of the earliest signs of CC [1]. It is associated with the severity of the disease. LT has been shown to reverse this anomaly in the majority of patients after 3 months of follow-up [8].

The purpose of the present study was to analyze the incidence of left ventricular DD in patients with cirrhosis and the impact on outcome during the peritransplantation period.

\section{METHODS}

We conducted a retrospective analysis of all adult patients undergoing LT for cirrhosis from January 2012 to June 2015 in the Centro Hospitalar e Universitário de Coimbra, Portugal. We excluded pediatric patients, liver retransplantation, and severe heart disease not correlated with cirrhosis (pacemaker or severe valve disorders in pretransplantation echocardiogram).

Analysis included demographic characteristics (age, sex), etiology of cirrhosis, Child-Pugh and Model for End-Stage Liver Disease (MELD) scores, corrected QT (QTc) interval in preoperative ECG, existence of DD and respective type, existence of systolic dysfunction and ejection fraction (EF) in pretransplantation echocardiography, mortality, duration of mechanical ventilation in the postoperative period, number and duration of vasopressor support used in the postoperative period.

We considered long QTc to be present when the value of the QTc interval was $>440 \mathrm{~ms}$. The QTc interval was calculated by means of the Fridericia formula. The echocardiographic criteria used for definition of DD were E/A ratio (corrected for age), prolonged deceleration time $(>200 \mathrm{~ms})$, prolonged isovolumetric relaxation time ( $>80 \mathrm{~ms}$ ), tissue Doppler measurements sampled at the level of the mitral annulus over the septal and lateral wall (annular $\mathrm{E}^{\prime}$ and $\mathrm{E} / \mathrm{E}^{\prime}$ lateral ratio), and enlarged left atria.

Statistical analysis was performed with the use of SPSS Statistics 20 with percentages for qualitative variables, and mean \pm SD or median for quantitative variables, depending on normality. To assess if the variables had a normal distribution, we used the Kolmogorov-Smirnov test. Statistical significance was determined with the chi-square test or Fisher for categoric independent variables; for numeric independent variables ( 2 groups) we used the Student $t$ test when they had a normal distribution and the MannWhitney $U$ test for samples without normal distribution. For numeric independent variables ( $>2$ groups) we used one-way analysis of variance test for samples with normal distribution and the Kruskal-Wallis test for samples without normal distribution. Statistical significance was assumed with $P<.05$.

\section{RESULTS}

The study included 106 patients undergoing LT for liver cirrhosis from January 2012 to June 2015; 80.2\% (85 patients) were male. The overall average age was 54.83 years (SD $8.52 \mathrm{y}$ ), with a minimum age of 30 years and the maximum age of 69 years.

In patients transplanted, the commonest etiology was alcoholic cirrhosis $(64.8 \%)$, followed by nonalcoholic causes (Table 1). At the time of transplantation, $43.4 \%$ of patients had hepatocellular carcinoma.

The median MELD score in our group of patients was 16 , with a minimum of 6 and maximum of 45 .

Child-Pugh class A was found in $21.6 \%$ of patients, class $\mathrm{B}$ in $23.0 \%$, and class $\mathrm{C}$ in $55.4 \%$.

The presence of a prolonged QTc interval in pretransplantation ECG was found in $19 \%$ of patients (20 patients).

In the pretransplantation echocardiography the existence of systolic dysfunction was found in $1.1 \%$ of the patients. Median EF was $64 \%$, with a minimum of $50 \%$ and maximum of $87 \%$.

DD was present in $35.8 \%$ of patients (38 patients), type 1 dysfunction was present in $71.1 \%$ of the cases, $26.3 \%$ of the cases had type $2 \mathrm{DD}$, and $2.6 \%$ of patients had type 3 .

Median mechanical ventilation was 10.0 hours after transplantation, with a minimum of 0 and a maximum of 199 hours.

In $6 \%$ of patients there was no use of postoperative vasopressor support, $74 \%$ of patients required 2 amines, $5 \%$ required 3 amines, and $15 \%$ only had 1 amine used in the postoperative period. The median of usage was 2 amines (minimum 0, maximum 3).

Vasopressor support was necessary for a median of 31 hours, with a minimum of 0 and a maximum of 197 hours.

Seventeen patients died during the study period (16\%). The hospital mortality rate, at 90 days after transplantation, was $4.72 \%$. The 1 -year survival was $86 \%$, the 2 -year survival was $81.3 \%$, and the 3-year survival was $78.2 \%$.

The presence of pretransplantation DD did not differ significantly with sex, etiology of cirrhosis, MELD ChildPugh or score, ventilation time, vasopressor support, or mortality (Table 2). The age and the existence of DD varied

Table 1. Etiology of Nonalcoholic Cirrhosis

\begin{tabular}{lc}
\hline \multicolumn{1}{c}{ Cause } & Incidence (\%) \\
\hline Primary biliary cirrhosis & 30.0 \\
Hepatitis B & 23.33 \\
Hepatitis C & 16.67 \\
Autoimmune hepatitis & 13.33 \\
Primary sclerosing cholangitis & 10.0 \\
Cryptogenic cirrhosis & 3.33 \\
Secondary biliary cirrhosis & 3.33 \\
\hline
\end{tabular}


Table 2. Correlation of Pretransplantation QTc or Diastolic Dysfunction (DD) With Demographic Characteristics, Etiology, Gravity Scores, Ventilation Time, Vasopressor Support, and Mortality

\begin{tabular}{lcc}
\hline \multicolumn{1}{c}{ Variable } & QTc & Diastolic Dysfunction \\
\hline Age & $P=.861$ & $P<.001$ \\
Sex & $P=.112$ & $P=.500$ \\
Etiology & $P=.344$ & $P=.584$ \\
MELD & $P=.922$ & $P=.389$ \\
Child-Pugh & $P=.286$ & $P=.077$ \\
Ventilation time & $P=.695$ & $P=.243$ \\
Vasopressor support & $P=.735$ & $P=.610$ \\
Mortality & $P=.184$ & $P=.051$ \\
\hline
\end{tabular}

Abbreviation: MELD, Model for End-Stage Liver Disease.

significantly $(P<.001)$, with a higher incidence of diastolic dysfunction increasing with age. The type of DD and the MELD score did not vary significantly.

We performed a logistic regression with mortality as the dependent variable and DD, prolonged QTc, age, and Child-Pugh and MELD scores as the factors, resulting in an explanatory power of $13.6 \%$ (Nagelkerke $R 2$ ), with DD as a significant factor with $P=.016$. We performed a multivariate analysis that excluded age as a confounder between DD and mortality.

The existence of prolonged QTc before transplantation did not correlate with statistical significance with the ventilation time, vasopressor support, or mortality (Table 2).

\section{DISCUSSION}

Our results, in a population of liver transplant patients due to cirrhosis of different etiologies, show that DD is fairly common in this setting and unrelated to the etiology of liver cirrhosis or MELD and Child-Pugh scores, but correlated with mortality.

Despite the increased basal cardiac output in cirrhotic patients, the cardiac response to physiologic and pharmacologic stimuli is decreased. $\mathrm{CC}$ does not show overt heart failure at rest owing to decreased afterload because of reduced peripheral vascular resistance. Stress conditions, including exercise, infection, drugs, hemorrhage, and any procedure, such as insertion of TIPS or LT, can precipitate the latent form of $\mathrm{CC}$ into overt heart failure. CC-related heart failure is claimed to be the 3rd leading cause of death after rejection and infection in transplant patients [9].

In our study, left ventricular EF was normal in almost all of the patients in the resting state, with just $1.1 \%$ of patients with $\mathrm{EF}<55 \%$. Therefore, the use of EF to evaluate systolic function has several limitations. In one study, the authors evaluated left ventricular systolic function by means of the peak systolic longitudinal strain [10]. They found that patients with cirrhosis had reduced longitudinal left ventricular systolic function, despite still having normal EF, and this was independent from etiology or clinical status.

Some reports have suggested that $\mathrm{CC}$ could be diagnosed with simple echocardiographic DD indexes, such as E/A ratio, even at rest [9]. In our study, although the prevalence of DD was relatively high $(35.8 \%)$, in most cases it was mild and unrelated to sex or etiology of cirrhosis. There was a correlation of DD with increasing age. However, other studies did not find this relationship, and DD was unrelated to age $[6,11]$. Also, it does not correlate with MELD and Child-Pugh scores. In agreement with our findings, studies have shown no correlation between DD and severity of liver dysfunction $[6,10]$. However, there is 1 study where DD was directly correlated with liver failure, with Child-Pugh and MELD scores significantly higher in patients with DD [11]. Although it did not differ statistically from mortality, with the chi-square test it approximated the correlation. When a logistic regression was performed, this correlation was revealed as significant, independently from age.

The QT interval represents the length of ventricular electric systole, and its prolongation may provide a substrate for ventricular arrhythmias or sudden death [12]. In some studies, ECG abnormalities were found to be associated with patient outcomes following LT, with a prolonged QTc interval being a predictor of peritransplantation heart failure and linked to lower posttransplantation survival [13]. However, in our study we did not find such a correlation.

There are some studies that report a prolonged QTc interval to be present in up to $50 \%$ of patients with cirrhosis, which is not related to the etiology of liver disease, worsens in parallel with the severity of disease, and may be associated with reduced survival [7]. In the present study, the prevalence of prolonged QTc interval was 19\%, lower than the prevalence reported in literature. That can be explained by 2 factors: In the majority of studies the formula that is used to calculate the QTc is the Bazett formula, which overcorrects the QT interval, increasing QTc at heart rates $>60$ beats/min, and that fact could be relevant in patients with cirrhosis, who usually have sinus tachycardia. There is 1 study that derives a specific "cirrhosis formula" that is very similar to the Fridericia formula [14]. Another possible reason is that, a great proportion of our patients (43.4\%) had hepatocellular carcinoma, and are proposed to LT in an early course of the disease compared with the others without hepatocellular carcinoma.

In this study, no differences were found between the clinical indexes of chronic liver disease (MELD and ChildPugh scores) and QTc prolongation in the preoperative period. Some studies show that QTc interval prolongation in cirrhotic patients correlates with Child-Pugh class B and C [12]. Also, we did not find any correlation between preoperative prolonged QTc and the duration of ventilation after LT or the need of vasopressor support or mortality.

Certain limitations should be considered when interpreting the results of the present study. It was conducted retrospectively, resulting in a few cases of missing data that could not be retrieved from patient records, and has a relatively small sample inherent to the number of transplantations. Our findings would need to be confirmed with the use of large multicenter studies. We propose a prospective study, including more patients and correlating also 
the incidence of QTc interval with the presence of adverse cardiac events, such as arrhythmias in the perioperative period, and mortality. Also, we suggest correlating the incidence of DD and prolonged QTc in the preoperative period with the presence of adverse cardiac events and mortality.

In conclusion, the prevalence of DD is relatively high in patients with cirrhosis, being in most cases of mild severity and unrelated to etiology of cirrhosis or MELD and ChildPugh scores. It seems to be an important factor conditioning mortality in cirrhotic patients undergoing LT. Although there are some studies that associate a prolonged QTc interval with reduced survival, in our study we did not find such a correlation.

\section{REFERENCES}

[1] Zardi EM, Zardi DM, Chin D, et al. Cirrhotic cardiomyopathy in the pre- and post-liver transplantation phase. J Cardiol 2016;67:125-30.

[2] Moller S, Bernardi M. Interactions of the heart and the liver. Eur Heart J 2013;34:2804-11.

[3] Fourlas CA, Alexopoulou AA. Cirrhotic cardiomyopathy. Hellenic J Cardiol 2004;45:114-20.

[4] Chayanupatkul M, Liangpunsakul S. Cirrhotic cardiomyopathy: review of pathophysiology and treatment. Hepatol Int 2014;8: 308-15.
[5] Karagiannakis DS, Papatheodoridis G, Vlachogiannakos J. Recent advances in cirrhotic cardiomyopathy. Dig Dis Sci 2015;60: $1141-51$.

[6] Somani PO, Contractor Q, Chaurasia AS, et al. Diastolic dysfunction characterizes cirrhotic cardiomyopathy. Indian Heart J 2014;66:649-55.

[7] Rahman S, Mallett SV. Cirrhotic cardiomyopathy: implications for the perioperative management of liver transplant patients. World J Hepatol 2015;7:507-20.

[8] Zamirian M, Tavassoli M, Aghasadeghi K. Corrected QT Interval and QT dispersion in cirrhotic patients before and after liver transplantation. Arch Iran Med 2012;15:375-7.

[9] Moaref A, Zamirian M, Yazdani M, et al. The correlation between echocardiographic findings and QT interval in cirrhotic patients. Int Cardiovasc Res J 2014;8:39-43.

[10] Sampaio F, Pimenta J, Bettencourt N, et al. Systolic and diastolic dysfunction in cirrhosis: a tissue-Doppler and speckle tracking echocardiography study. Liver Int 2013;33:1158-65.

[11] Nazar A, Guevara M, Sitges M, et al. Left ventricular function assessed by echocardiography in cirrhosis: relationship to systemic hemodynamics and renal dysfunction. J Hepatol 2013;58:51-7.

[12] Mimidis K, Papadopoulos V, Thomopoulos K, et al. Prolongation of the QTc interval in patients with cirrhosis. Ann Gastroenterol 2003;16:155-8.

[13] Josefsson A, Fu M, Bjornsson E, et al. Prevalence of pretransplant electrocardiographic abnormalities and post-transplant cardiac events in patients with liver cirrhosis. BMC Gastroenterol $2014 ; 14: 65$

[14] Zambruni A, Micoli AD, Lubisco A, et al. QT Interval correction in patients with cirrhosis. J Cardiovasc Electrophysiol 2007; 18:77-82. 